\title{
Outcome of non-transected anastmoticurethroplasty for management of short bulbar urethral stricture
}

\author{
MostafaMahmoud,atefgalal,essamsallem,ahmedriad
}

\begin{abstract}
Excision and end-to-end anastomosis (EPA) has been the preferred urethroplasty technique for short bulbar strictures and is associated with an excellent functional outcome. Driven by concerns over the potential morbidity associated with dividing the urethra, therefore compromising spongiosal blood flow, as well as spongiofibrosis being superficial in the majority of non-traumatic bulbar strictures, the nontransecting technique for bulbar urethroplasty has been developed with the aim of achieving the same success as EPA without the morbidity associated with transection. This manuscript highlights the fundamentalprinciples underlying the ongoing debatetransection or non-transection of the strictured bulbar urethra? The potential advantages of avoiding dividing the corpus spongiosum of the urethra are discussed. The non-transecting anastomotic procedure together with its various modifications aredecribed in detail. Our experience with this technique is presented. Non-transecting excision of spongiofibrosis with preservation of well vascularised underlying spongiosum provides an excellent alternative to dividing the urethra during urethroplasty for short non-traumatic proximal bulbar strictre
\end{abstract}

\section{Introduction}

The optimal management of bulbar urethral stricture continues to generate much debate within the reconstructive The optimal management of bulbar urethral strictures continues to generate much debate within the reconstructive urological community; endoscopic intervention (dilatation or urethrotomy) $v s$. urethroplasty, flap vs. graft, dorsal or ventral augmentation, just to mention a few of the controversial issues commonly discussed. Most recently the question of whether to transect the bulbar urethra or not in non-traumatic strictures has been raised (1). The surgical treatment of bulbar urethral strictures guided by stricture aetiology, length, location and previous surgical intervention (2). Traditionally the first and simplest form of treatment has been endoscopic instrumentation by dilatation or urethrotomy in the hope that healing by secondary intention will occur before further scarring. The cure rate is only around $60 \%$, and this only in short strictures of the bulbar urethra (3). The long-term success rate and cost effectiveness decreases even further with subsequent attempts at endoscopic intervention (4). Therefore, in patients with bulbar strictures which are refractory to endoscopic manouvres, urethroplasty remains the only curative option (5). The bulbar urethra is the commonest site for urethral strictures in the developed world. Most are idiopathic, occurring most frequently at the junction of the proximal and middle thirds. Short, sharp strictures in this location may indeed be congenital in origin due to failure of complete canalisation of the urogenital membrane $(6,7)$. Other common causes of bulbar urethral stricture are iatrogenic (usually 
following traumatic catheterisation or urethral instrumentation during TURP) and fall-astride perineal injuries. The current literature suggests that when such strictures are short, the best form of urethroplasty is excision of the strictured urethral segment (spongiofibrosis and surrounding corpus spongiosum) and tension-free end-to-end anastomosis of the healthy spatulated edges (EPA) $(8,9)$. Traditionally, strictures shorter than 1$2 \mathrm{~cm}$ are considered

to be amenable to EPA due to concerns regarding tension

Transection vs. non-transection for short bulbar

\section{strictures}

Transection of the urethra for short bulbar strictures was originally challenged at the 2009 American Urological Association (AUA) meeting (10). The debate in favour or against transection with end-to-end anastomosis and any non-transecting technique is based on stricture aetiology and the success rates and complications associated with each treatment modality.

In 2007, Jordan described a modification to EPA in which the proximal urethral blood supply is preserved (11). In proximal bulbar strictures the bulbar arteries are mobilized and preserved. The strictured area is excised and the corpus spongiosum reconstituted over the anastomosis. This technique is not appropriate in strictures with significant spongiofibrosis. We have independently developed the technique of nontransectinganastomotic bulbar urethroplasty with the aim of achieving the same functional outcome as EPA while avoiding the potential morbidity associated with divisionof the spongiosum and bulbar arteries

Table 1 Transecting vs. nontransecting techniques

Transecting the urethra
- Allows complete removal of scar tissue reducing risk of

restricture

- $>95 \%$ success rate in the long term

- But may cause vascular damage to the urethra

- But is associated with increased sexual dysfunction

Non-transecting the urethra

- Prevents complete removal of scar tissue leading to

- stricture recurrence

- Up to $85 \%$ success rate which deteriorates over time

- But preserves urethral vasculature

But therefore causes less sexual dysfunction

Surgical technique-non-transecting bulbar urethroplasty

All patients were manged with the non-transected uretheroplasty technique as described by Mundey et al2012

Preoperatively Prophylactic antibiotics (cefotaxieme) are administered in a dose of $1 \mathrm{gm}$ IV adminsterd 1 hour before surgery. Spinal anasthezea was used in all patients and the procedure was performed while the patients in lithotomy position. A midline perineal incision was done extending from the perineo-scrotal junction to about $1 \mathrm{~cm}$ from the anal margin. The incession was deepened through the subcutaneous tissue and Colles' fascia to expose the bulbospongiosus muscle. A plane was created between the colles fascia and the underlying bulbar urethra by opening Gallaudet's fascia. The bulbospogiosus muscle was then divided in the midline and retracted. The bulbar urethra is dissected off the tunica albuginea of the corpora dorsally. A catheter was then passed along the urethra till the distal end of the stricture and a dorsal stricturotomy was made that extended distally and proximally till'normal' - caliber urethra on either ends was reached. 
The fibrotic spongiosal tissue on the ventral aspect of the stricture segment was then exised. The longtidunally incised uretheral mucosa was then transversely closed with interrupted $5 / 0$ polygyclolic acid sutures and with the knots on the inside. The repair is done on 18 fr catheter. The collesfascia ,bulbospongiosus muscle ,subcutenous tissue were then anatomically closed with placement of a suction drain to be left for 48 hs. Post-operatively, the patient started oral feeding 6hours after surgery and received postoperative antibiotics in form of fortum $1 \mathrm{gm}$ twice daily for 2 days and parentral analgesics in form of ketolac on demand. The patiets were discharged after removal of drain and received oral antibiotics for $10 \mathrm{ds} .(12)$

\section{Patient and methods}

This is A clinical non-randamised prospective study that included 55 patients presented to urological department of sohaguniverisity hospital during the period from 1may 2015 to 31decemper 2017. The patients presented with stricture bulbar urethera that was due to falling astride in 20 cases and non-traumatic causes in 35 patients. Those 35 cases with non-traumatic stricture were investigated by VCUG, sonourethrographey to assess the length of stricture segment and degree of spongiofibrosi. Out of those patients five cases were excluded due to (long stricture segment in 4 cases and dense spongiofibrosi in one case). The reminanning 30 cases were included in the study as they met our inclusion criteria which are
1- short bulbar stricture urethera ranging from $<2 \mathrm{~cm}$ in length

2- No past history suggesting urethral trauma or repeated VIU

3- The patients' age ranges from 17-55 year

4- Mild spongiofibrosis as assesd by sonouretherographey.

All patients were evaluated preoperatively by urine analysis, culture and sensitivity,routine lab investigations. The patients were evaluated clinicaly by history taking, physical examination with assessment of IPSS (international prostate symptoms score) to assess the obstructive voiding symptoms and assessment of IIEF (international index of erectile function) for evaluation of potency. Uroflowmetry was done for all patients with recording maximal flow rate (Qmax).The aime of our work is to evaluate the outcome on nontransected anastmoticuretheroplasty technique on the patency of the urethera and the potency of the patient. The catheter was left for 2 weks and the patients were then evaluated at $1,3,6,9$ and 12 months postoperatively .

During each visit, the patient was evaluated by history taking with assesmnet of IPSS and IIEF with recording of maximal flow rate by uroflowmetry. The integrity of the repair was aslo delineated by VCUG and AUG at each visit.

Success was defined as normal postoperative uretherogram with improved IPSS and no significant change in the IIEF score.

\section{Results}

The study included 30 patients with short bulbar stricture urethra with mean age 35.47 years, with SD 13.1 and median duration of symptoms of 2 years

\section{Causes of bulbar stricture urethra and associated morbidities:}

Stricture bulbar urethra was diagnosed based upon VCUG, RUG, sonouretherographey.

Causes of Bulbar stricture urethra was post-inflammatory stricture in 22 patients 
SOHAG MEDICAL JOURNAL Outcome of non-transected anastmoticurethroplasty Vol. 23 No.2 Apr 2019

$(62.3 \%)$, and it was iatrogenic stricture in 8 patients $(38.75 \%)$

All patients were managed with non-transecting anastomotic uretheroplasty and the procedure was completed as planned in all of them. The average operation time was 65.67 minutes, with SD 16.491 while the average blood loss was $78.83 \mathrm{ml}$, SD 23.3. None of our patients required blood transfusion.

Post operatively:

Good outcome was encounter in 28 patient with iumproved voiding parameters. The post-operative voiding parameters are demonstrated in (table 6)

\begin{tabular}{|c|c|c|c|}
\hline Parameter & $\begin{array}{c}\text { Pre-uretheroplasty } \\
\text { (median\& } \\
\text { IQR) }\end{array}$ & $\begin{array}{c}\text { Post-uretheroplasty } \\
\text { (median \& IQR) }\end{array}$ & P value \\
Uroflowmetry data & 320 (IQR: $225-431)$ & $65($ IQR: $0-105)$ & \\
PVR (ml) & $7.37($ IQR: $0-10.5)$ & $26.2($ IQR: $15-28)$ & \\
Q-max (ml/sec) & 13.7 & 6.1 & \\
\hline IPSS & 19 & 18 & \\
\hline IIEF & & & \\
\hline
\end{tabular}

Table 6: Improvement in the clinical data after non-transecting urethroplasty.

Early post operative complications: On studying the post-operative complications all patients werer conservatively managed

-Wound infection was recorded in four cases that needed antibiotic theraby.

-urinary tract infection was noticed in 3 cases that needed culture and sensitivity then antibiotics

-scrotal swelling, scrotal edema was noticed in 6 patients that needed treatment with alpha-chemotripsin

-Recurent stricture e encounterd on follow up and thae were managed with VIU.

\section{Discussion}

The anatomy of the urethra, particularly the bulbomembranous urethra, provides the basis for the nontransecting technique: the dorsal aspect of the bulbomembranous urethra is devoid of corpus spongiosum; it has a concave configuration as it curves around the point of fusion of the corporal bodies; it can stretch considerably as occurs when the bladder is displaced upwards by an expanding pelvic haematoma following a pelvic fracture. The first property means that a dorsal stricturotomy is performed in a relatively avascular plane preserving the spongiosal blood flow ventrally.
The second and third provide sufficient length to allow closure of a longitudinal stricturotomy in horizonal plane without tension.(Sugimoto et al. 2005). In principle, this technique of longitudinal stricturotomy and transverse closure is a form of Heinecke-Mickuliczstricturoplasty, best known in intestinal surgery while The original idea of avoiding urethral transection in proximal bulbar urethroplasty came from G.H).In our study of 30 patients we show asuccess rate of $93.3 \%$ which is more than many other series and comparable with the other techniques with more excellent postoperative results and patients satisfaction. 
As regard the mean age of our study group was 35.47 years, with SD 13.1, this was similar to results of Ofoha et al. (2015) in his study of anastomotic Urethroplasty for Short Segment Bulbar Urethral Stricture as the mean age of the patients in his study was 38.9 years which is a common age for stricture urethra as stricture urethra were uncommon in those less than 20 years and above 70 years old. Tijani et al.(2009) recorded a mean age of 43.1 years, which is similar to the findings of other investigators and our study.

Mean of operative time in our study was 65.67 minutes, with SD 16.491, and our mean of blood loss was 78. 83 $\mathrm{ml}, \mathrm{SD}$ 23.3. Wei Le et al in his study of total 23 patints treated by nontransecting anastomotic bulbomembranousurethroplasty show amean operative time $128.50 \pm 12.15$ min and blood loss $316.67 \pm 64.99 \mathrm{ml}$ (Wei Le et al 2017) which is much more than ours and this can be explained by the fact that etiology of stricture in his patients were due to traumatic posterior urethral stricture with massive adhesions and scar tissue around.

Mean stricture length in our study was $1.75 \mathrm{~cm}$, with SD $0.25 \mathrm{~cm}$, and this was similar to results of Bugeja et al. (2015) for non-transecting bulbar urethroplasty as the mean stricture length in their patients group was 1.6 centimetres, also in Ofoha et al. (2015) study found that the mean stricture length was $1.6 \mathrm{~cm}$ with a range of $0.5 \mathrm{~cm}$ to $2.5 \mathrm{~cm}$, this was shorter than that in results of Bugeja et al. (2016) asmean stricture length in their study was $5.3 \mathrm{~cm}$ and this may be due to the use of another techinique of non-transecting bulbar urethroplasty using buccal mucosa. (Range 3-8 cm). In our study the mean value of preoperative Q max was $7.37 \mathrm{ml} / \mathrm{sec}$, while postoperative was $26.2 \mathrm{ml} / \mathrm{sec}$, We found that $\mathrm{Q}$ max on uroflowetry was significantly increased after nontransecting bulbar urethroplasty suggesting functional improvement associated with the procedure, Pavone et al in his study of 27 patients underwent urethroplasty surgery using dorsal buccal mucosa graft show apreoperative mean of Q $\max 7.4 \mathrm{ml} / \mathrm{s}$ and postoperative mean about 21.16 $\mathrm{ml} / \mathrm{s}$ (Pavone et al 2017) so we are more or less have a better results with our technique.Lumen et al in his study of Twenty-one patients treated for stricture urethra using differnt techniques as anastomotic repair, freegraft urethroplasty, combined urethroplasty and perineostomy show marked improvement in IPSS and IIEF, in his study the mean preoperative IPSS was 15.8 which improve to reach 6.4 postoperative, while the mean of the IIEF was 15 preoperative and postoperative became 11.6 (lumen et al 2011). In a systematic review, Blaschkoet al. (2013) evaluated sexual function following urethroplasty, and noted de novo erectile dysfunction in $0-38 \%$ of patients. Similarly, in a comparative review of sexual-related side effects between anastomotic and buccal graft substitution urethroplasty, Palminteri et al. (2010) reported higher rates of penile curvature, shortening, impaired erection, and overall sexual dissatisfaction in patients undergoing EPA. However, the results of these reviews were limited by a lack of standardized sexual function questionnaire and limited information on comorbid conditions that impact erectile function (Blaschko et al. 2013).Non transecting urethroplasty leads to a significant improvement in urinary flow and IPSS, Urinary continence is tending to improve, mainly due to postoperative amelioration of urge, urgeincontinence and postvoid dribbling. And no significant erectile dysfunction 
was noted after non transecting urethroplasty. Patient satisfaction after urethroplasty was high and not related to absence of recurrence. Functional outcome should be assessed after urethroplasty.

However the limitations of our study was the short term follow up, the small number of patients and this study was single center experience. So studies with more patients and a longer follow-up are needed to assess the long-term functional outcome of such chalengeing technique

\section{Conclusions}

Stricture aetiology is the main determinant of whether transection of the bulbar urethra is necessary or not. It is inevitable in trauma when the fibrosis must be completely excised and healthy urethral edges anastomosed to secure the most durable result. It is however certainly not necessary in short non-traumatic proximal bulbar strictures. A dorsal approach to these strictures coupled with a nontransecting technique prevents disruption of the integrity of ventral spongiosal blood flow and there are practical and theoretical reasons for doing so. Functional outcomes are equivalent to transection and end-to-end anastomosis with less surgical trauma. Minimising surgical trauma and preservation of blood supply are key principles for any surgical intervention, so The nontransecting anastomotic bulbar urethroplasty technique used appears to give results that are as good as those of traditional anastomotic urethroplsty.

\section{References}

1. Ahmed A, Kalayi GD. Urethral stricture at Ahmadu Bello University Teaching Hospital, Zaria.EastAfr Med J. 1998; 75(10):582- 585.

2. Al-Qudah HS, Santucci RA (2005) Extended complications of urethroplasty. IntBraz J Urol 31:315325.

3. Al-Qudah HS, Santucci RA. Buccal mucosal onlayurethroplasty versus anastomotic urethroplasty (AU) for short urethral strictures: which is better? J Urol 2006;175:103.

4. Alwaal A, Blaschko SD, McAninch JW, Breyer BN. Epidemiology of urethral strictures. TranslAndrolUrol 2014; 3(2):209-213.

5. Andrich DE, Dunglison N, Greenwell TJ et al (2003) The long-term results of urethroplasty. J Urol 170(1):90-92.

6. Andrich DE, Leach CJ, Mundy AR. The Barbagli procedure gives the best results for patch urethroplasty of the bulbar urethra. BJU Int 2001;88:385-9.

7. Andrich DE, Mundy AR. Complications of social lithotomy. BJU Int 2010;106:40-1.

8. Andrich DE, Mundy AR. Nontransecting anastomotic bulbar urethroplasty: a preliminary report. BJU Int 2012;109:1090-4.

9. Andrich DE, Mundy AR. What is the best technique for urethroplasty? EurUrol 2008;54:1031-41.

10.Anger JT, Buckley JC, Santucci RA, Elliott SP, Saigal CS: Trends in stricture management among male Medicare beneficiaries: under - use of urethroplasty? Urology 2011; 77: 4815.

11. Atak $M$, et al. Low-power holmium:YAG laser urethrotomy for urethral stricture disease: comparison of outcomes with the cold-knife technique. Kaohsiung J. Med. Sci. 2011;27:503-507.

12.Barbagli G, De Angelis M, Romano G, et al. Long-term followup of bulbar end-to-end anastomosis: a retrospective analysis of 153 patients in a single center experience. J Urol 2007;178:2470-3.

13.Barbagli G, et al. Bulbar urethroplasty using buccal mucosa grafts placed on the ventral, dorsal or lateral surface of the urethra: are results affected by the surgical technique? J. Urol. 2005;174:955-958.

14. Barbagli G, Guazzoni G, Lazzeri M. One-stage bulbar urethroplasty: 
retrospective analysis of the results in 375 patients. EurUrol 2008;53:828-33.

15.Barbagli G, Palminteri E, Lazzeri M, Turini D. Interim outcomes of dorsal skin graft bulbar urethroplasty. J. Urol. 2004;172:1365-1367.

16.Barbagli G, Perovic S, Djinovic R, Sansalone S, Lazzeri M. Retrospective descriptive analysis of 1,176 patients with failed hypospadias repair. J. Urol. 2010;183:207-211.

17.Barbagli G, Sansalone S, Djinovic R, Romano G, Lazzeri M. Current controversies in reconstructive surgery of the anterior urethra: a clinical overview. Int. Braz. J. Urol. 2012;38:307-316.

18. Baskin LS, McAninch JW (1993) Childhood urethral injuries: perspectives on outcome and treatment. Br J Urol 72:241-246. 